\title{
Summation and configuration between and within sensory modalities in classical conditioning of the rabbit
}

\author{
E. JAMES KEHOE, AMANDA J. HORNE, PETER S. HORNE, and MICHAELA MACRAE \\ University of New South Wales, Sydney, New South Wales, Australia
}

\begin{abstract}
Three experiments examined "atomistic" and "configural" processes in stimulus compounding using the rabbit's conditioned nictitating membrane response. Two conditioned stimuli (CSs) were trained separately and then tested together in a compound. Animals trained with CSs from different modalities-namely, tone and light-showed summation in both acquisition and extinction. That is, the probability of a response to the compound could be predicted by the statistical sum of responding to the CSs. In contrast, animals trained with CSs from the auditory modality, tone and noise, showed a level of responding to the tone + noise compound that was the same as that of the CSs, well under the level predicted by the statistical sum of responding to the CSs. In conclusion, atomistic processes appear to predominate in cross-modal compounding. Configural processes may occur during compounding within the auditory modality, but atomistic alternativesnamely, common elements and selective attention hypotheses-may be able to explain the results.
\end{abstract}

The present experiments were conducted to determine the contribution of atomistic and configural processes in stimulus compounding. Two conditioned stimuli (CSs) were trained separately and were then tested together in a compound. CSs from both the same and different sensory modalities were used. Two complimentary hypotheses were tested: (1) that atomistic processes are stronger than configural processes when the CSs are from different modalities, and (2) that configural processes predominate when the CSs are from the same modality.

The usual outcome of stimulus compounding is summation, meaning that the compound yields a higher level of responding than do the individual CSs (see, e.g., Couvillon \& Bitterman, 1982; Kehoe \& Graham, 1988; Weiss, 1972, 1978). However, for theoretical purposes, it is not clear what is being summated. According to an atomistic hypothesis, the summation outcome reflects the summation of the associative strengths of the CSs (Kehoe \& Graham, 1988; Weiss, 1972). In contrast, configural hypotheses contend that compounding the CSs produces a perceptual interaction in the encoding of the CSs. Most commonly, this interaction is seen as a fusion that supplants the individual CSs (Heinemann \& Chase, 1975; Pearce, 1987). Less commonly, the perceptual interaction is seen as a mutual alteration in the encoding of the CSs (Hull, 1943; Meehl, 1945; see also Kehoe \& Gormezano, 1980). In either case, responding to the compound stimulus reflects the summation of generalized associative

Preparation of this manuscript was supported by Australian Research Council Grants AC89322441 and AC9231222. Correspondence should be sent to E. J. Kehoe, School of Psychology, University of New South Wales, P.O. Box 1, Kensington, NSW 2033, Australia (e-mail: jimk@ hydra.maths.unsw.edu.au). strengths from the encodings of the separate CSs. With sufficient generalization, responding to the compound could exceed responding to the CSs.

As may be apparent, the summation outcome can be explained by atomistic and configural hypotheses. There are, however, two sets of results that appear to be more decisive, but in opposite ways. On the one hand, in conditioning of the rabbit's nictitating membrane (NM) response, the quantitative character of stimulus compounding with tone and light CSs has favored an atomistic hypothesis. On the other hand, similar research in autoshaping of the pigeon has pointed toward a configural hypothesis.

With regard to the rabbit NM results, the quantitative relation among the levels of responding to the compound and its CSs follows a precise but simple formula. Specifically, the percentage conditioned responses (CRs) to the test compound $(\mathrm{Pc})$ can be predicted by the sum of the percentage CRs to the separate CSs $(\mathrm{Pa}, \mathrm{Pb})$ as combined by the formula for statistically independent events, namely, $\mathrm{Pa}+\mathrm{Pb}-(\mathrm{Pa} \times \mathrm{Pb}$ ) (Kehoe, 1982b, 1986; Kehoe \& Graham, 1988). The correlation between the actual and predicted responding to the compound has ranged from .68 to .86 , with perhaps a $5 \%$ underprediction. This close correspondence is consistent with an atomistic hypothesis and not a configural hypothesis. Unless there were complete generalization from the CSs to the configural stimulus, a pure configural hypothesis would expect the mathematically summated levels of responding to overpredict the observed level of responding to the compound.

With regard to autoshaping of the pigeon (Pearce \& Wilson, 1991), extinction was conducted with the compound after initial training with the individual CSs. Tests 
with the CSs revealed that they produced more responding than the compound did. This outcome is consistent with a configural hypothesis, in that extinction of responding to the novel compound did not fully generalize back to the CSs. In contrast, an atomistic hypothesis predicts that responding to the CSs should have remained below that of the compound.

This large discrepancy in the results from different preparations indicates that atomistic and configural processes may be engaged during stimulus compounding in a variable fashion. Numerous factors, of course, could be responsible for the discrepancy. Among them, however, there is reason to suppose that the modality of the CSs may determine whether atomistic or configural processes are engaged in a test compound. The exact summation seen in the rabbit NM preparation has been obtained with the use of stimuli from different modalities-namely, auditory and visual. In contrast, the configural results in autoshaping were obtained with the use of stimuli from the same sensory modality-namely, colored triangles and their spatial location. Although these stimuli are highly distinctive, configuration may still occur more readily within a modality than between modalities.

In a study of latent inhibition, Honey and Hall (1989) have provided indirect evidence that stimuli combine differently, depending on whether they are from the same or a different modality. Specifically, Honey and Hall preexposed rats to a compound of either tone + light or tone + clicker. A third group of rats was given preexposure to the tone only. Then, in a conditioned suppression procedure, all rats were given pairings of the tone with a shock unconditioned stimulus (US). The rats given preexposure to the tone showed slow acquisition of suppression to the tone. The rats given preexposure to the tone + light compound also showed slow acquisition, which may have been even slower than that of the tone-alone group. The rats given preexposure to the tone + clicker, however, showed more rapid acquisition-that is, less latent inhibition.

To explain these results, Honey and Hall (1989) proposed a configural hypothesis. Specifically, they contended that the perception of the tone was relatively unaffected by the light but was altered by the clicker. Accordingly, the deleterious effects of preexposure generalized readily from the tone + light compound to tone-US training. However, preexposure to the tone + clicker compound generalized weakly to tone-US training.

Conditioning theories largely treat stimuli in an indifferent fashion as far as their modalities are concerned. Atomistic theories assume that stimuli always retain their specific perceptual characters when they are combined (e.g., Kehoe, 1988; Rescorla \& Wagner, 1972; Weiss, 1972, 1978). Conversely, in configural theories, it is assumed that a perceptual fusion or interaction always occurs when stimuli are combined (see, e.g., Hull, 1943; Pearce, 1987). As a simplifying assumption, this indifference to stimulus modality can be useful but misleading, particularly where conclusions about the atomistic versus configural processes begin to diverge. From a broader perspective, there is good reason to believe that the modalities of combined stimuli may matter. Stimuli from the same modality have the opportunity to interact beginning at the receptor surface, whereas stimuli from different modalities can interact only at a later step in their neural processing. Accordingly, the present experiments were conducted to determine whether stimuli from one modality-namely, the auditory modality - would combine in a different way than stimuli from two modalities-namely, the auditory plus visual modalities.

\section{EXPERIMENT 1}

Experiment 1 provided a within-subjects comparison of stimulus compounding between and within modalities. Thus, all subjects received training with three CSs--tone, light, and noise-and they were tested with the three possible compounds during acquisition training. Following acquisition, responding in half the subjects was extinguished using the tone + light compound, and responding in the other half was extinguished with the tone + noise compound.

\section{Method}

Subjects. The subjects were 16 naive female albino rabbits (Oryctolagus cuniculus), weighing approximately $1.5 \mathrm{~kg}$ on arrival from the university's breeding unit. All rabbits had free access to food and water in their home cages.

Apparatus. The apparatus and recording procedure for the NM response were patterned after those of Gormezano (1966) and are detailed by Kehoe, Feyer, and Moses (1981). The rabbits were trained in one of eight sound-attenuating chambers. On the wall of the chamber in front of each subject was a stimulus panel, and behind the subject, an exhaust fan. A speaker was mounted at the midpoint of the stimulus panel, $8 \mathrm{~cm}$ anterior to and $16 \mathrm{~cm}$ above the rabbit's head. The speaker provided two auditory CSs, a 1000$\mathrm{Hz}, 84-\mathrm{dB}$ (SPL) tone and an 84-dB white noise. Both were $800 \mathrm{msec}$ in duration and were superimposed on an ambient noise level of $81 \mathrm{~dB}$ provided by the exhaust fans. Mounted on the stimulus panel, $4 \mathrm{~cm}$ above the speaker, was an $8-\mathrm{W}$ neon houselight. To provide a visual CS, the houselight was flashed at $20 \mathrm{~Hz}$ for $800 \mathrm{msec}$. The US was a $100-\mathrm{msec}, 3-\mathrm{mA}, 50-\mathrm{Hz}$ ac electric current delivered via 9-mm stainless steel Autoclip wound clips positioned $10 \mathrm{~mm}$ apart and $15 \mathrm{~mm}$ posterior to the lateral canthus of the rabbit's right eye. On CS-US trials, the interval between CS onset and US onset was $800 \mathrm{msec}$. The sequence and timing of stimulus events were controlled by an Apple II computer equipped with interfaces and software developed by Scandrett and Gormezano (1980).

During training, each rabbit was restrained in a Perspex box (45 $\times 14 \times 22 \mathrm{~cm}$ ). The rabbit was held in place by the insertion of its head through an adjustable stock and by the securing of its ears to the front of the stock with a polyurethane foam-covered metal clamp. A muzzle-like head set, fitted securely about the snout, supported a photoelectric transducer for detecting movements of the NM (Gormezano \& Gibbs, 1988). A small, tinned copper wire hook was attached to a silk loop sutured in the NM of the rabbit's right eye. The other end of the hook contained a loop that fitted over the curved end of an L-shaped piano-wire crank, which operated 
the transducer. The signal from the transducer was amplified and transmitted to an analog/digital converter installed in the computer (10-msec sample rate).

Procedure. All rabbits received 1 day of preparation, 2 days of rest, and 1 day of adaptation before training began. On the day of preparation, hair posterior to the rabbit's right eye was removed, a small loop of silk (000 Dynex) was sutured into the NM of the right eye, and the rabbits were returned to their homes cages. On the adaptation day, the rabbits were placed in the conditioning apparatus for $60 \mathrm{~min}$, but no stimuli were presented.

Following adaptation, the subjects were split randomly into two groups $(n=8)$. Both groups first received 20 days of acquisition training. On each day, they received 20 tone-US trials $(\mathrm{T}+), 20$ light-US trials $(\mathrm{L}+)$, and 20 noise-US trials $(\mathrm{N}+)$. These training trials were intermixed so that no more than two of any one kind occurred in a row. In addition, every 11 th trial was a nonreinforced test trial for a compound stimulus. Each day, the subjects received two trials each of tone + light (TL), tone + noise (TN), and noise + light (NL).

At the end of acquisition training, both groups received 3 days of extinction. One group received extinction with the TL compound, and the other group received extinction with the TN compound. Each day contained 54 nonreinforced presentations of the appropriate compound. In addition, both groups received four nonreinforced test trials each of the tone, light, and noise. A test occurred on the 6th and 11 th trial of each sequence of 11 trials. In both stages of the experiment, the intertrial interval was $60 \mathrm{sec}$ (range, $50-70 \mathrm{sec}$ ).

Response definition. A CR was defined as any extension of the $\mathrm{NM}$ greater than or equal to $0.5 \mathrm{~mm}$ that occurred following the onset of the CS but prior to its termination. In extinction, the observation interval was extended to $1,200 \mathrm{msec}$ following CS onset.

\section{Results and Discussion}

Acquisition. Figure 1 shows the mean percent CRs across days of stimulus compounding during acquisition. Each panel depicts responding to one of the three compounds and their CSs. Inspection of Figure 1 reveals that summation appeared in the two cross-modal compounds, namely, TL and NL, but not in the auditory compound, TN. Specifically, the overall level of responding on TL trials ( $M=68 \% \mathrm{CRs}$ ) was significantly greater than the overall level of responding to either the tone ( $M=47 \%$ CRs) or the light $(M=44 \% \mathrm{CRs})[F \mathrm{~s}(1,14)=33.08$,
$35.28, p s<.01]$. Similarly, the level of responding to the NL compound ( $M=61 \% \mathrm{CRs)}$ was significantly greater than responding to either the noise $(M=41 \%$ CRs) or the light $(M=44 \%$ CRs $)[F \mathrm{~s}(1,14)=19.81$, $20.90, p s<.01]$. In contrast, the TN compound $(M=$ $46 \% \mathrm{CRs})$ produced a level of responding that failed to differ significantly from the levels of the tone $(M=47 \%$ CRs $)$ or noise $(M=41 \%$ CRs $)\left[F_{\mathrm{S}}(1,14)=0.22,1.67\right.$, ps $>.05]$.

The statistical summation rule was tested by applying it to the performance of each subject on each day. In agreement with previous findings (Kehoe, 1982b, 1986; Kehoe \& Graham, 1988), responding to the cross-modal compounds was only slightly underpredicted by the statistical sum of responding to the CSs. Specifically, responding on TL trials $(M=68 \% \mathrm{CRs})$ was slightly greater than the summated responding to tone and light ( $M=61 \% \mathrm{CRs})$. This difference approached but did not reach statistical significance $[F(1,14)=3.99, p<.10]$. Responding on NL trials $(M=61 \% \mathrm{CRs})$ was even more closely approximated by summated responding to the noise and light $(M=$ $58 \%$ CRs) $[F(1,14)=1.47, p>.10]$.

To determine how well the predictions from the summated CSs agreed with actual responding to the compound, a correlation coefficient was calculated, with each subject's predicted and actual responding averaged over all days of acquisition. These correlation coefficients confirmed the correspondence seen in the group means. Specifically, the subject-by-subject correlations between responding for the compound and the summated CSs for the TL and NL compounds were .83 and .89 ( $p$ s $<.01$, $N=16$ ).

The application of the statistical rule for the TN compound revealed that its responding was vastly overpredicted by the summated responding to the CSs. Responding on TN trials ( $M=46 \% \mathrm{CRs}$ ) was much less than the summated responding to the tone and noise $(M=$ $60 \%$ CRs) $[F(1,14)=51.00, p<.01]$. Although there was a large constant error in the prediction, responding
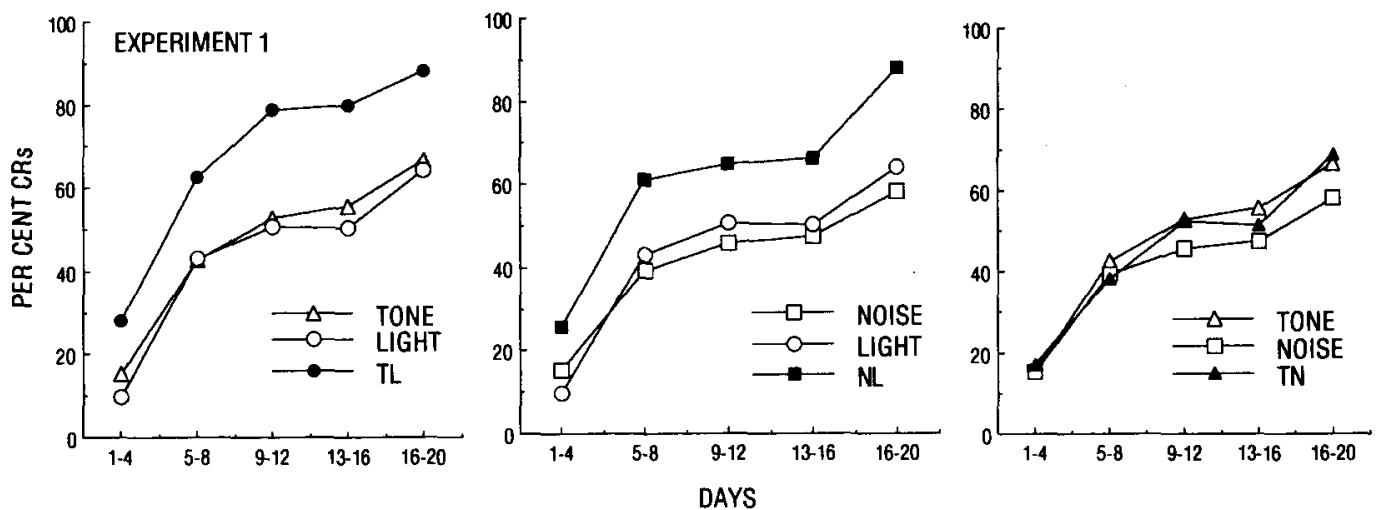

Figure 1. Mean percent conditioned responses during acquisition training in Experiment 1. Each panel depicts responding to one of the three compounds and its conditioned stimuli. TL, tone + light; NL, noise + light; TN, tone + noise. 
on the compound trials paralleled the summated responding to the CSs. The subject-by-subject correlation was high and significant $(r=.94, p<.01, N=16)$.

Extinction. Extinction was rapid in both groups. Responding to the compounds and the individual CSs largely ceased within the 1st day of extinction. There was little spontaneous recovery. In what could be seen during the first block of 30 trials, the pattern of responding was similar to that seen in acquisition. That is, the level of responding on TL trials ( $M=31 \%$ CRs) was significantly higher than on either the tone $(M=6 \% \mathrm{CRs})$ or light $(M=0 \% \mathrm{CRs})$ trials $\left[F_{\mathrm{s}}(1,14)=7.42,7.93, p \mathrm{~s}<.01\right]$. There were similar levels of responding on trials for TN $(M=42 \%$ CRs $)$, T $(M=56 \% \mathrm{CRs})$, and N $(M=44 \%$ CRs), none of which differed significantly.

Summary. Experiment 1 revealed distinct differences in stimulus compounding depending on the modality of the CSs. During acquisition training, compounding across the auditory and visual modalities produced summation that conformed to the statistical summation formula. In contrast, compounding within the auditory modality did not yield summation. Instead, the level of responding to the TN compound was similar to the level of responding to the individual CSs.

\section{EXPERIMENT 2}

Experiment 2 was conducted with two aims in mind. First, it was a between-groups replication of Experiment 1 . Although summation across modalities is well established, the lack of summation within the auditory modality has not been reported previously for the rabbit NM preparation. Second, we wished to determine the effects of compounding a trained CS with an untrained stimulus. Pavlov (1927, p. 45) reported that such compounding produced "external inhibition"; that is, responding to the trained CS showed a decrease when the untrained stimulus was added to form the compound. This decrease is consistent with a configural hypothesis, because there would be incomplete generalization to the compound from the trained CS. Conversely, Pavlov's findings are inconsistent with an atomistic hypothesis. It would expect responding to the trained CS to be unaltered by the addition of a stimulus that has no associative strength.

To achieve these two aims, we trained the animals with two CSs and tested them with a third stimulus. Specifically, half the animals were trained with the tone and light CSs. Summation across modalities was tested with the TL compound. To identify any effects of compounding a trained CS with an untrained stimulus, the animals were also tested with a TN compound. The other half of the animals were trained with tone and noise. Hence, for them, tests with the TN compound examined the combination of trained stimuli within the auditory modality, and tests with the TL compound examined the combination of trained and untrained stimuli across modalities.

\section{Method}

Unless otherwise indicated, the apparatus and procedures were identical to those used in Experiment 1 . Sixteen rabbits were assigned randomly to two groups $(n=8)$. One group, Group TL, received acquisition training with tone $(\mathrm{T}+)$ and light $(\mathrm{L}+)$. Following acquisition, that group received extinction using the TL compound. The other group, Group TN, received training with tone $(\mathrm{T}+)$ and noise $(\mathrm{N}+)$, after which responding was extinguished with the $\mathrm{TN}$ compound. Both groups received 10 days of acquisition training. Each day contained $30 \mathrm{~T}+$ trials, 30 trials of the other $\mathrm{CS}(\mathrm{L}+$ or $\mathrm{N}+$ ), $3 \mathrm{TL}-$ trials, and $3 \mathrm{TN}-$ trials. After acquisition was complete, extinction was run for 3 days. Each day contained 54 trials of the appropriate compound ( $\mathrm{TL}-$ or $\mathrm{TN}-$ ) plus 4 test trials each of tone, light, and noise.

\section{Results and Discussion}

One animal in Group TN died during the course of the experiment. Its data were eliminated from all analyses.

Acquisition. Figure 2 shows the mean percent CRs across days of stimulus compounding during acquisition training. The left-hand panel shows responding in Group TL. The right-hand panel shows responding in Group TN.

The results replicated those of Experiment 1. As can be seen in Figure 2, responding in Group TL showed cross-modal summation. That is, the level of responding on TL trials $(M=70 \%$ CRs) was significantly greater than that with either the tone ( $M=44 \%$ CRs) or the light $(M=54 \%$ CRs $)[F \mathrm{~s}(1,13)=37.35,10.27, p \mathrm{~s}<.01]$. In fact, there was oversummation; the statistical sum of responding to the tone and light $(M=61 \% \mathrm{CRs})$ significantly underpredicted responding on TL trials $(M=70 \%$ CRs) $[F(1,13)=11.29, p<.01]$. The subject-by-subject correlation was large and significant $(r=.98, p<.01$, $n=8$ ).

Responding in Group TN failed to show summation within the auditory modality. The level of responding on TN trials ( $M=55 \%$ CRs) was higher than that with the tone $(M=47 \%$ CRs $)[F(1,13)=5.14, p<.05]$, but was similar to that with the noise $(M=54 \%$ CRs $)(F<1)$. Not surprisingly, statistical summation of responding to tone and noise ( $M=63 \% \mathrm{CRs})$ significantly overpredicted responding on TN trials ( $M=55 \%$ CRs) $[F(1,13)=7.73, p<.05]$. Notwithstanding the overprediction, the subject-by-subject correlation showed a strong parallel between the summated responding and compound responding $(r=.94, p<.01, n=7)$.

In both groups, responding to the tone appeared unaffected when the tone was compounded with an untrained stimulus. In Group TL, responding on TN trials ( $M=$ $44 \%$ CRs) equaled that on T trials ( $M=44 \% \mathrm{CRs}$ ). Likewise, in Group TN, responding on TL trials ( $M=48 \%$ CRs) virtually equaled that on T trials ( $M=47 \% \mathrm{CRs})$. Any apparent differences were not significant $\left(F_{S}<1\right)$.

Extinction. In agreement with the results of Experiment 1 , responding in extinction dropped rapidly, but, before disappearing, it showed the same pattern of respond- 

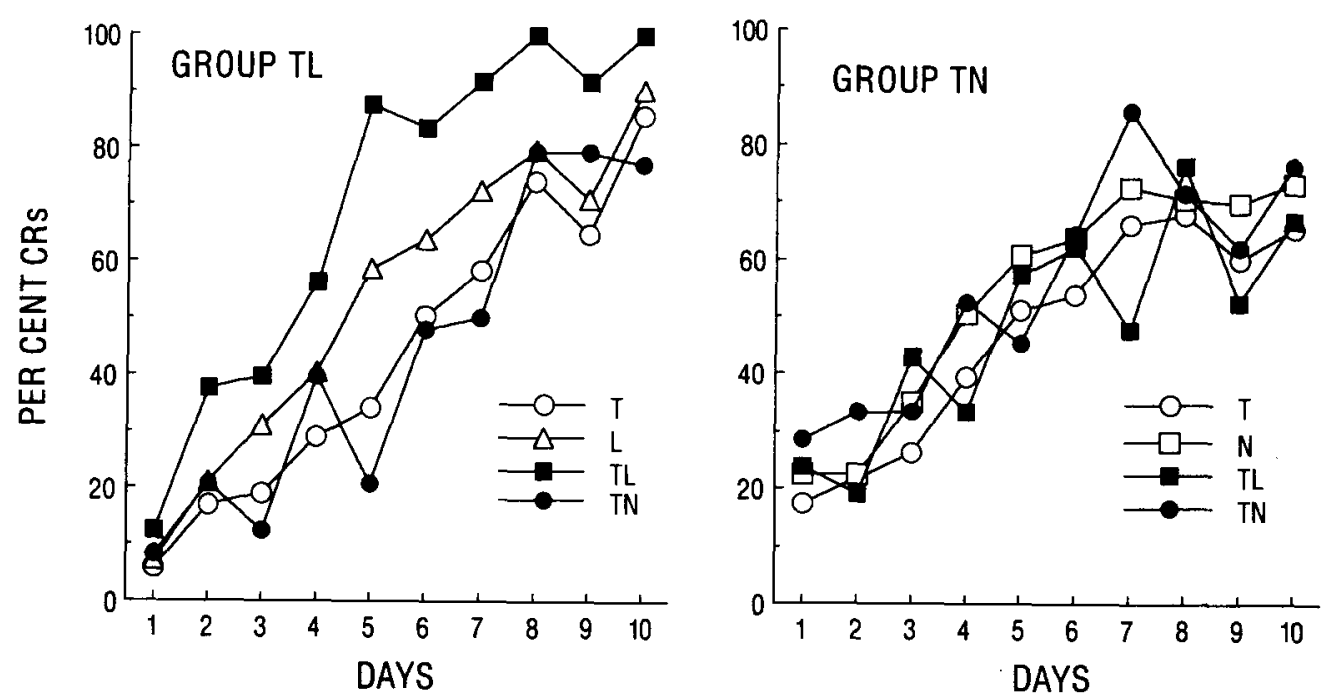

Figure 2. Mean percent conditioned responses during acquisition training in Experiment 2. Each panel depicts responding to the tone + light (TL) compound, the tone + noise (TN) compound, and the two trained conditioned stimuli in Groups TL and TN, respectively.

ing to the compound and CSs as that seen in acquisition. In the first block of 30 trials, Group TL showed a significantly higher level of responding on TL - trials $(M=$ $57 \% \mathrm{CRs})$ than on either the tone ( $M=25 \% \mathrm{CRs})$ or light $(M=25 \% \mathrm{CRs})$ trials $[F \mathrm{~s}(1,13)=11.56,21.09$, $p s<.01]$. Responding in Group TN extinguished so rapidly that there was a floor effect in the first block of trials. Responding on TN - trials ( $M=14 \% \mathrm{CRs})$ did not differ significantly from responding to either the tone ( $M=0 \%$ CRs) or the noise $(M=21 \%$ CRs).

\section{EXPERIMENT 3}

The results of Experiments 1 and 2 for acquisition were clear, but the low level of responding in extinction made it difficult to discern differences between the compounds and the CSs. Accordingly, Experiment 3 was aimed at raising the level of responding in acquisition and later in extinction. Specifically, in Experiment 3 we used a shorter, more optimal CS-US interval in acquisition and fewer trials per session in extinction.

\section{Method}

Unless otherwise indicated, the apparatus and procedures were identical to those in Experiment 2. The subjects were 16 rabbits assigned randomly to two groups $(n=8)$. One group was again labeled as Group TL. It received acquisition training with tone (T+) and light $(\mathrm{L}+)$, followed by extinction with the TL compound. The other group was labeled $\mathrm{TN}$, received training with tone $(\mathrm{T}+)$ and noise $(\mathrm{N}+)$, followed by extinction with the TN compound. Both groups received 8 days of acquisition training and 5 days of extinction. Each day of extinction contained 20 trials of the appropriate compound ( $\mathrm{TL}-$ or $\mathrm{TN}-$ ) plus two test trials each of tone and either light or noise. The CS duration throughout the experiment was $400 \mathrm{msec}$, and, in acquisition, the CS-US interval was $400 \mathrm{msec}$.

\section{Results and Discussion}

Acquisition. Figure 3 shows the mean percent CRs during acquisition training. As seen in Experiments 1 and 2 , Group TL showed cross-modal summation. That is, the level of responding on TL trials ( $M=77 \%$ CRs) was significantly greater than that with either the tone $(M=$ $62 \% \mathrm{CRs})$ or the light $(M=66 \% \mathrm{CRs})\left[F_{\mathrm{s}}(1,14)=\right.$ $22.77,14.29, p s<.01]$. The statistical summation of responding to the tone and light ( $M=75 \% \mathrm{CRs})$ only slightly underpredicted responding on TL trials $(M=77 \%$ CRs) $(F<1)$. The subject-by-subject correlation was nearly perfect $(r=.99, p<.01, n=8)$.

In Group TN, the level of responding on TN trials ( $M=64 \%$ CRs) was higher than that of the tone $(M=$ $57 \%$ CRs) $[F(1,14)=2.40, p>.10]$ and was slightly lower than that of the noise $(M=67 \% \mathrm{CRs})(F<1)$. Statistical summation of responding to tone and noise ( $M=72 \%$ CRs) significantly overpredicted responding on TN trials $(M=64 \%$ CRs) $[F(1,13)=6.15, p<.05]$. The subject-by-subject correlation demonstrated a strong parallel between the summated responding and compound responding $(r=.93, p<.01, n=8)$.

As seen in Experiment 2, responding to tone was unaffected when it was compounded with an untrained stimulus. In Group TL, responding on TN trials $(M=60 \%$ CRs) was only marginally less than that on T trials $(M=$ $62 \% \mathrm{CRs}$ ). In Group TN, responding on TL trials ( $M=$ $65 \% \mathrm{CRs}$ ) was in fact significantly greater than that on $\mathrm{T}$ trials $(M=57 \% \mathrm{CRs})[F(1,14)=6.23, p<.05]$.

Extinction. Figure 4 shows the mean levels of responding to the compound and the individual CSs across all 5 days of extinction. With only 20 rather than 60 compound trials per session, extinction was spread over 5 sessions. As was seen in Experiments 1 and 2, Group TL showed 

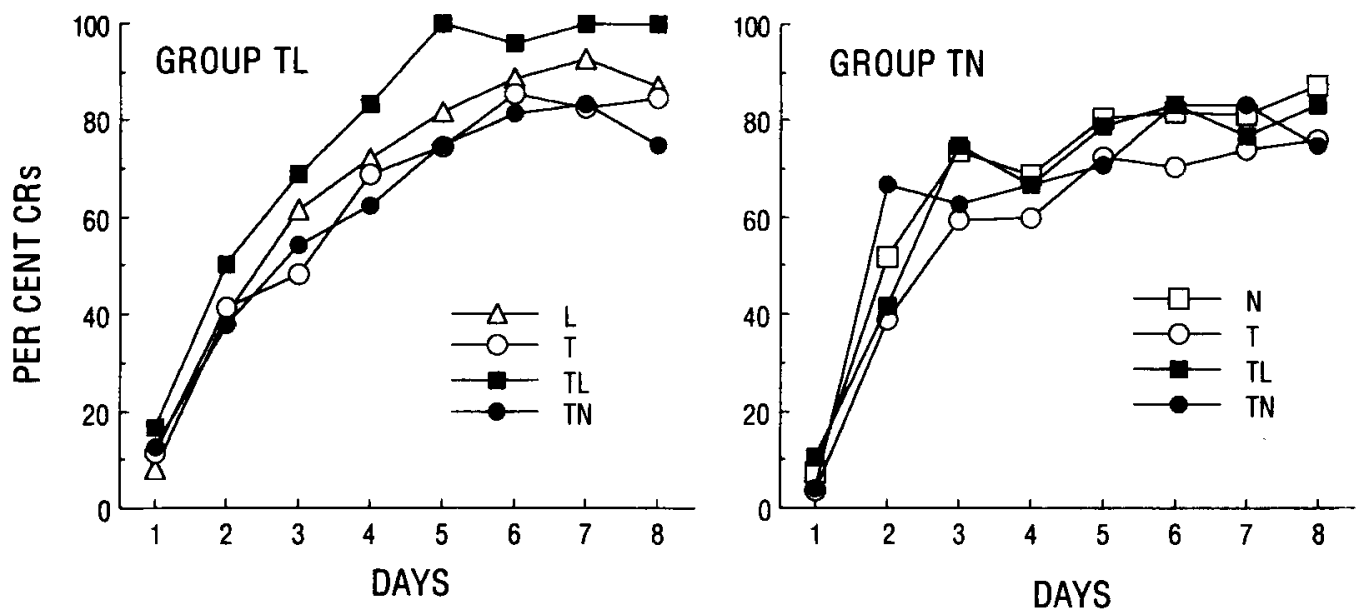

Figure 3. Mean percent conditioned responses during acquisition training in Experiment 3. Each panel depicts responding to the tone + light (TL) compound, the tone + noise (TN) compound, and the two trained conditioned stimuli in Groups TL and TN, respectively.

summation. Specifically, in the first block of 20 trials, Group TL showed a higher level of responding on TL trials $(M=80 \% \mathrm{CRs})$ than on either the tone $(M=31 \%$ CRs) or the light $(M=56 \% \mathrm{CRs})$ trials $[F \mathrm{~s}(1,14)=$ $12.73,7.30, p s<.05]$. As can be seen in Figure 4, the same pattern appeared over all 5 days, the overall means on TL, T, and L trials were $40 \%, 18 \%$, and $24 \% \mathrm{CRs,}$ respectively. In contrast, Group TN showed no evidence of summation. In the first block of 20 trials, Group TN showed a level of responding on TN trials $(M=69 \%$ CRs) that was between those of tone ( $M=50 \%$ CRs) and noise $(M=75 \% \mathrm{CRs})$. However, the apparent differences were not significant $[F \mathrm{~s}(1,14)=2.04,<1, p s>$ .10]. This pattern appeared throughout extinction but did not achieve significance. The overall means on TN, T, and $N$ trials were $36 \%, 28 \%$, and $43 \%$ CRs, respectively.

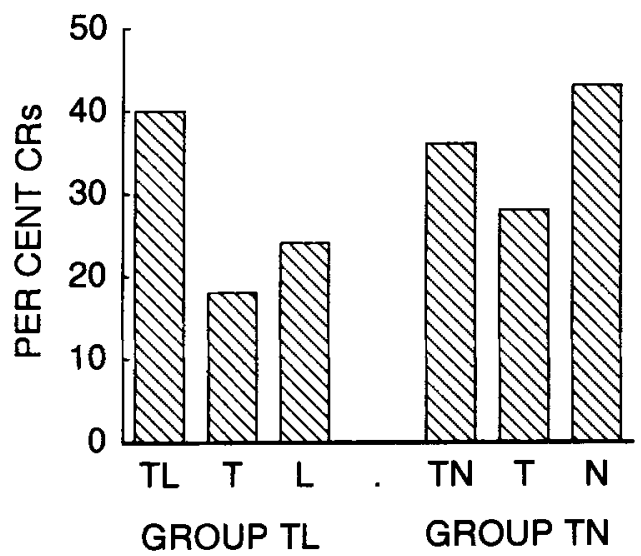

Figure 4. Mean percent conditioned responses during extinction in Experiment 3. The figure shows the overall mean level of responding on tone + light (TL), tone (T), and light (L) trials in Group TL and on tone + noise (TN), tone, and noise (N) trials in Group TN.
The statistical summation rule was applied to responding across extinction. For the TL compound ( $M=40 \% \mathrm{CRs),}$ the summation of responding to tone and light yielded a significant underprediction $(M=31 \% \mathrm{CRs})[F(1,14)=$ $5.49, p<.05]$. For the TN compound $(M=36 \% \mathrm{CRs})$, the summation rule yielded a significant overprediction $(M=51 \%$ CRs $)[F(1,14)=13.50, p<.01]$

\section{GENERAL DISCUSSION}

All three experiments yielded the following major findings: (1) In agreement with previous examinations of cross-modal stimulus compounding, animals trained with the tone and light showed summation in both acquisition and extinction. That is, responding to a tone + light compound was greater than responding to either single stimulus. (2) Moreover, the level of responding to the tone + light compound conformed to the statistical sum of responding to the CSs (Kehoe \& Graham, 1988; Kehoe \& Schreurs, 1986). Where there were inaccuracies, responding to the compound tended to be greater than would be expected by statistical summation. (3) In contrast to the cross-modal results, animals trained with tone and noise showed a level of responding to the compound that was equal to that for the CSs. (4) The statistical sum of the responding to the CSs overpredicted the level of responding to the auditory compound.

At first blush, the interpretation of the present results appears easy. On the one hand, auditory and visual stimuli appear to combine in an atomistic fashion. On the other hand, different auditory stimuli appear to combine in a configural fashion, through either perceptual fusion or mutual interaction. However, other aspects of the present results and other closely related findings suggest that this interpretation must be approached with caution.

Notwithstanding cross-modal summation, it cannot be inferred that the combination of auditory and visual stimuli is always atomistic. There is abundant evidence that tone 
+ light compounds can engage configural processes (Kehoe, 1982a, 1986; Kehoe \& Graham, 1988; Kehoe \& Schreurs, 1986). For example, Kehoe and Graham followed stimulus compounding with training using a "negative patterning" schedule. In the negative patterning schedule, frequent $\mathrm{TL}-$ trials were interspersed among $\mathrm{T}+$ trials and $\mathrm{L}+$ trials. Gradually, responding to the TL compound overcame summation, declined, and dropped below responding to either the tone or the light. The suppression of responding to the compound cannot be explained by summation of the excitatory values of the CSs. Instead, the compound appears to engage a configural stimulus, which, in turn, acquires an inhibitory value that counteracts summation (see Kehoe \& Graham, 1988; Rescorla, 1973; Woodbury, 1942).

With regard to the compounding of auditory stimuli, it might seem that they would interact in an innate, perceptual manner. That is, the perceptual interaction would depend only on the joint presentation of the two stimuli regardless of their associative strengths. If such a hypothesis were true, the compounding of an established tone CS with the untrained noise CS should have produced a substantial drop in responding. This drop would reflect incomplete generalization from the $\mathrm{T}+$ trials to the $\mathrm{TN}$ test trials. Contrary to this hypothesis, there was no discernible decrease in responding when this manipulation was conducted in Experiments 2 and 3 . Hence, a perceptual interaction may have occurred within the auditory modality only when both the tone and the noise had acquired associative strength.

It is conceivable that only CSs with associative strength will engage configural processes within the auditory modality. However, current models of conditioning assume that perceptual encoding is preassociative in nature (e.g., Hull, 1943; Pearce, 1987; Rescorla \& Wagner, 1972). Accordingly, it is difficult to see how they would feed associative strength into the encoding process particularly for the purpose of predicting performance on a test trial. Beyond configural models, there are two types of atomistic hypotheses that may offer worthwhile explanations. They are the common elements hypotheses and attentional hypotheses.

A common elements hypothesis can explain the present results if it is assumed that each member of a pair of CSs comprises a set of distinctive elements and a set of shared elements (Kehoe \& Gormezano, 1980; Rescorla, 1972, 1973). Previous examinations of generalization in the rabbit NM preparation have shown that some elements would be shared by the tone and noise stimuli (Kehoe \& Napier, 1991). However, the light stimulus would share virtually no elements with the auditory stimuli (Kehoe \& Holt, 1984; Kehoe, Morrow, \& Holt, 1984). Accordingly, training with $\mathrm{T}+, \mathrm{N}+$, and $\mathrm{L}+$ would be construed as $\mathrm{TX}+, \mathrm{NX}+$, and $\mathrm{L}+$, where $\mathrm{X}$ represents the set of shared elements. Under this hypothesis, cross-modal compounding of, say, the tone with the light yields undiminished summation based on the associative strengths of T, X, and L. Compounding of the tone and noise, however, is another story. If the proportion of common ele- ments relative to the distinctive elements is high, then combined associative strengths of $\mathrm{T}, \mathrm{N}$, and $\mathrm{X}$ would be little greater than responding to TX or NX separately. Moreover, when the established tone CS was compounded with an untrained light or noise stimulus, the level of responding to TX would be unaffected.

Although the common elements hypothesis generally explains the present results, it encounters a stumbling block in that it predicts that the compounding of established tone and noise CSs should produce some summation unless there are very many shared elements and very few distinctive elements. Because there was little sign of summation when the tone and noise CSs were compounded, it would have to be inferred that the tone and noise had few if any distinctive elements. This inference, however, is contrary to other available data that tone and noise are readily distinguishable. First, there is only about $50 \%$ generalization from tones to white noise (Kehoe \& Napier, 1991). Second, the same tone and white noise used in this experiment have been used successfully in differential conditioning (Kehoe, Marshall-Goodell, \& Gormezano, 1987).

An attentional hypothesis can explain the present results if the total channel capacity for two modalities is greater than it is for one modality. Thus, for tone and light, the associative strengths would add together in an unhindered fashion. For tone and noise, however, competition between them for the smaller channel capacity would preclude full summation of their respective associative strengths. In the simplest case, it could be assumed that the stimulus with the strongest associative strength determines response output (cf. Sutherland \& Mackintosh, 1971 , p. 39). If so, no summation within the modality would occur. That is, responding on a TN compound trial would be about the same as that to a separate tone CS and/or noise CS. Likewise, responding to the established tone CS would not be affected by the addition of the untrained noise stimulus.

Although an attentional hypothesis can explain the present results, it would be difficult to extend to it some other closely related results. In particular, it has long been recognized that attentional hypotheses have difficulty in explaining explicit differentiation between a compound and its components - as, for example, in negative patterning (Mackintosh, 1975; Sutherland \& Mackintosh, 1971). Such a differentiation requires simultaneous attention to two stimuli to permit their integration in some fashion. Where there is adequate channel capacity, as is the case with the tone + light compound, there is no problem in explaining negative patterning. However, where there is a small channel capacity, as may be the case for the tone + noise, there should be little opportunity for integration of the two stimuli. If this hypothesis is correct, negative patterning may be difficult to achieve within the auditory modality. As far as can be found, this surprising deduction is not correct. Pavlov (1927, p. 144) makes a passing mention that negative patterning could be easily achieved with stimuli in the "same analyser." More to the point, Rescorla $(1972,1973)$ successfully demon- 
strated negative patterning with the use of a tone + clicker compound. Moreover, when tone + clicker and tone + light compounds were used for purposes of counterbalancing, Rescorla (1973) did not report any difference in the rate at which negative patterning appeared.

In conclusion, the present findings can be viewed on two levels. On one level, they roughly confirm the original hypotheses. That is, for the rabbit NM preparation, atomistic processes clearly predominate in cross-modal compounding, and other processes evidence themselves during compounding within the auditory modality. At the second level, however, it is not clear what the other processes may be. Among the available alternatives, no single hypothesis appears to be entirely satisfactory. For the configural hypothesis, responding to the established tone CS was not discernibly reduced by the addition of an untrained noise stimulus. For the common elements hypothesis, some modest summation failed to appear when the tone CS and noise CS were compounded. In these two cases, the difficulty arises because a predicted difference failed to occur. Accordingly, the usual caution must be exercised in accepting a no-difference finding. The same caution must be exercised in accepting the attentional explanation of the same null findings.

Although it would have been convenient if the results had clearly pointed to one hypothesis, they do provide two more general lessons. First, the examination of compounding and other related procedures with the use of purely auditory stimuli may be a rich source of information concerning the nature of complex stimulus processing. Second, just as some response systems are particularly sensitive to certain CS-US combinations (e.g., taste and toxin), responding to different CS-CS combinations may be prone to different processes of integration.

\section{REFERENCES}

Couvillon, P. A., \& Bitterman, M. E. (1982). Compound conditioning in honeybees. Journal of Comparative \& Physiological Psychology, 96, 192-199.

Gormezano, I. (1966). Classical conditioning. In J. B. Sidowski (Ed.), Experimental methods and instrumentation in psychology (pp. 385420). New York: McGraw-Hill.

Gormezano, I., \& GibBs, C. M. (1988). Transduction of the rabbit's nictitating membrane response. Behavior Research Methods, Instruments, \& Computers, 20, 18-21.

HeinemanN, E. G., \& Chase, S. (1975). Stimulus generalization. In W. K. Estes (Eds.), Handbook of learning and cognitive processes (pp. 305-349). Hillsdale, NJ: Erlbaum.

HONEY, R. C., \& HALL, G. (1989). Attenuation of latent inhibition after compound pre-exposure: Associative and perceptual explanations. Quarterly Journal of Experimental Psychology, 41B, 355-368.

Hull, C. L. (1943). Principles of behavior. New York: AppletonCentury-Crofts.

KEHOE, E. J. (1982a). Conditioning with serial compound stimuli: Theoretical and empirical issues. Experimental Animal Behaviour, 1, 30-65.

KEHOE, E. J. (1982b). Overshadowing and summation in compound stimulus conditioning of the rabbit's nictitating membrane response. Journal of Experimental Psychology: Animal Behavior Processes, 8, 313-328.

KEHOE, E. J. (1986). Summation and configuration in conditioning of the rabbit's nictitating membrane response to compound stimuli. Jour- nal of Experimental Psychology: Animal Behavior Processes, 12, 186-195.

KEHOE, E. J. (1988). A layered network model of associative learning: Learning-to-learn and configuration. Psychological Review, 95, 411-433.

Kehoe, E. J., Feyer, A., \& Moses, J. L. (1981). Second-order conditioning of the rabbit's nictitating membrane response as a function of the CS2-CS1 and CS1-US intervals. Animal Learning \& Behavior, 9, 304-315.

Kenoe, E. J., \& Gormezano, I. (1980). Configuration and combination laws in conditioning with compound stimuli. Psychological Bulletin, 87, 351-378.

Kehoe, E. J., \& Graham, P. (1988). Summation and configuration in negative patterning of the rabbit's conditioned nictitating membrane response. Journal of Experimental Psychology: Animal Behavior Processes, 14, 320-333.

KeHOE, E. J., \& HoLT, P. E. (1984). Transfer across CS-US intervals and sensory modalities in classical conditioning in the rabbit. Animal Learning \& Behavior, 12, 122-128.

Kehoe, E. J., Marshall-Goodell, B., \& Gormezano, I. (1987). Differential conditioning of the rabbit's nictitating membrane response to serial compound stimuli. Journal of Experimental Psychology: Animal Behavior Processes, 13, 17-30.

Kehoe, E. J., Morrow, L. D., \& Holt, P. E. (1984). General transfer across sensory modalities survives reductions in the original conditioned reflex in the rabbit. Animal Learning \& Behavior, 12, 129-136.

KeHOE, E. J., \& NAPIER, R. M. (1991). Real-time factors in the rabbit's nictitating membrane response to pulsed and serial conditioned stimuli. Animal Learning \& Behavior, 19, 195-206.

KEHOE, E. J., \& SCHREURS, B. G. (1986). Compound conditioning of the rabbit's nictitating membrane response: Test trial manipulations. Bulletin of the Psychonomic Society, 24, 79-81.

Mackintosh, N. J. (1975). A theory of attention: Variation in the associability of stimuli with reinforcement. Psychological Review, 82, 276-298.

MeEHL, P. E. (1945). An examination of the treatment of stimulus patterning in Professor Hull's "Principles of Behavior." Psychological Review, 52, 324-332.

Pavlov, I. P. (1927). Conditioned reflexes (G. V. Anrep, Trans.). London: Oxford University Press.

Pearce, J. M. (1987). A model for stimulus generalization in Pavlovian conditioning. Psychological Review, 94, 61-73.

Pearce, J. M., \& WiLson, P. N. (1991). Effects of extinction with a compound conditioned stimulus. Journal of Experimental Psychology: Animal Behavior Processes, 17, 151-162.

ResCoRla, R. A. (1972). "Configural" conditioning in discrete-trial barpressing. Journal of Comparative \& Physiological Psychology, 79, 307-317.

Rescorla, R. A. (1973). Evidence for "unique stimulus" account of configural conditioning. Journal of Comparative \& Physiological Psychology, 85, 331-338.

Rescorla, R. A., \& Wagner, A. R. (1972). A theory of Pavlovian conditioning: Variations in the effectiveness of reinforcement and nonreinforcement. In A. H. Black \& W. F. Prokasy (Eds.), Classical conditioning II (pp. 64-99). New York: Appleton-Century-Crofts.

SCANDRETT, J., \& GoRMEZANO, I. (1980). Microprocessor control and A/D data acquisition in classical conditioning. Behavior Research Methods \& Instrumentation, 12, 120-125.

SUTHERLAND, N. S., \& MaCKINTOSH, N. J. (1971). Mechanisms of animal discrimination learning. New York: Academic Press.

Weiss, S. J. (1972). Stimulus compounding in free operant and classical conditioning: A review and analysis. Psychological Bulletin, 78, 189-208.

WEISS, S. J. (1978). Discriminated response and incentive processes in operant conditioning: A two-factor model of stimulus control. Journal of the Experimental Analysis of Behavior, 3, 361-381.

WoodBury, C. B. (1942). The learning of stimulus patterns in dogs. Journal of Comparative Psychology, 35, 29-40.

(Manuscript received January 3, 1993; revision accepted for publication May 21, 1993.) 